\title{
Herbal medicine use and its associated factors among pregnant women attending antenatal care at Wollega University Referral Hospital, West Ethiopia.
}

Busha Gamachu Labata ( $\sim$ bushagemechu1@gmail.com )

Wollega University https://orcid.org/0000-0001-8170-7232

Getu Bayisa Wakjira

Wollega University

Mohammed Gebre Dedefo

Wollega University

Ginenus Fekadu Mekonen

Wollega University

Eba Abdisa Golja

Wollega University

Dessalegn Dereje Jaleta

Ethiopian Institute of Agricultural Research

Research

Keywords: Associated factor, Herbal medicine, Pregnant, Women

Posted Date: January 7th, 2020

DOI: https://doi.org/10.21203/rs.2.20123/v1

License: (9) (i) This work is licensed under a Creative Commons Attribution 4.0 International License.

Read Full License 


\section{Abstract}

Background: Herbal medications are plant-derived materials or products with therapeutic or other human health benefits, which contain either raw or processed ingredients from one or more plants. There are many different types of herbal medicine that spring from different cultures around the world. All these have the use of medicinal plants in common. Developing world general population relies on these traditional medicines for primary care. Besides, pregnant women use herbal medicines because of pregnancy related ailments and normal aliments. Therefore, the study aimed to assess prevalence and factors associated with herbal medicine use among pregnant women attending antenatal care at Wollega University Referral Hospital.

Method: A facility based cross sectional study was conducted among pregnant women attending Wollega University Referral Hospital antenatal care from May to June 2018. Written informed consent was obtained from study participants prior to data collection. Univariate and Multivariate logistic regression analysis was used to determine factors associated with herbal medicine use. Association was significant when p-value is less than 0.05 .

Result: A total of 238 pregnant mothers were participated in the study. The respondents' age ranges from 17 to 46 years. The prevalence of herbal medicine use among pregnant women attending antenatal care at Wollega University Referral Hospital was $66.40 \%$. The use of herbal medicine during pregnancy was 3.885 times more likely among rural residents $(A O R=3.885,95 \% \mathrm{Cl} 1.121-13.47)$ than urban residents. Respondents who has monthly income between 1001 to 2000 Ethiopian Birr (AOR=2.585, 95\% Cl 1.1255.940) were 2.585 times more likely to use Herbal medicine as compared to those who earn $>3000$ Ethiopian Birr. However, mothers of planned pregnancy ( $A O R=0.335,95 \% \mathrm{Cl} 0.114-0.986$ ) were less likely to use Herbal medicine as compared to those who had unplanned pregnancy.

Conclusion: The prevalence of herbal medicine use among pregnant women attending antenatal care at Wollega University Referral hospital was $66.40 \%$. Commonly used herbs were Garlic $(24.37 \%)$ and ginger (18.07\%). Rural residence and low income had significantly associated to herbal medicine use while planned pregnancy was inversely associated to herbal medicine use.

\section{Background}

Herbal medications are Botanical or Phytotherapy, which were plant-derived materials or products with therapeutic or other human health benefits, which contain either raw or processed ingredients from one or more plants. In some traditions, materials of inorganic or animal origin may also be present $(1,2)$. There are many different types of herbal medicine that spring from different cultures around the world. All these have the use of medicinal plants in common, but they vary in the plants they use, the way they prepare and use medicines from these plants, and the philosophy of their treatment approaches. Different cultures may also use the same plants but differ in how it is used, or the part they use (3-5). 
Herbal medicines were used in every country around the world and $70-95 \%$ of developing world general population rely on these traditional medicines for primary care $(6,7)$. Besides, herbal medicines use among pregnant women was also common. In this population group use of herbal medicine varies from $22.3 \%$ to $82.3 \%$ in middle East, $48.6 \%$ in Gondar Ethiopia and $73.1 \%$ in Hosanna Ethiopia $(3,4,8)$. The majority of studies reported maximum use of herbs were common during the first trimester of pregnancy probably due to the higher incidence of pregnancy related problems during this period $(3,9)$. Pregnant women who use herbal medicine agree that herbal medicine was safe during pregnancy and efficacious over prescribed drugs for some illnesses and condition (10).

Herbal medicine has both beneficial and potential side effect. Herbs contain antioxidants, essential oils, and vitamins, which fight against germs, toxins, boost immunity level and have anti-inflammatory function (2). In addition, herbal medicine use cause abdominal pain, rash and Chronic renal failure $(2,11,12)$.

Pregnant women use herbal medicines to treat pregnancy related ailments and normal aliments $(10,13)$. The pregnant women, in the preparation of the medicines for their diseases and conditions, used parts of raw medicinal plants. They used leaves, roots, barks, fruits, seeds and whole plant (13). Besides, they also use aniseed, fenugreek, ginger, garlic, green tea, peppermint, eucalyptus, tenaadam, damakesse, feto and omore $(8,14)$. These herbal medicine were mostly indicated for the management of toothache, various types of pain, flu, malaria, respiratory tract infections, digestive problems, inducing labour, skin problems, nausea, vomiting and pregnancy related conditions such as swollen feet and back pain $(10,15)$.

Therefore, the aim of this study is to assess prevalence of herbal medicine use and its associated factors in pregnancy women.

\section{Methods}

\section{Study area and period}

The study was conducted in Wollega University Referral Hospital (WURH) antenatal care (ANC), which is located in Nekemte Town, Oromia region, West Ethiopia. The study was conducted from May to June 2018 at ANC.

\section{Study design}

A facility based cross sectional study was conducted

\section{Study population}

All pregnant women attending ANC at WURH during the study period were included in the study. However, those pregnant women who were critically ill and unable to hear and speak were excluded from study. 


\section{Sample Size Determination and Sampling Technique}

The sample size was calculated using formula for a single population proportion considering the following assumptions: A 95\% confidence level, margin of error (0.05) and proportion of herbal medicine use $50.4 \%(p=0.504)(16)$. Then substituting these values in the single population proportion formula yields 384 pregnant mothers.

$\mathrm{n}=\underline{\mathrm{Z}} \underline{\underline{2}}-(\underline{1}-\mathrm{p})-\mathrm{p} \quad=(\underline{1.96}) \underline{\underline{2}}-(\underline{1-0.504}) * \underline{0.504}=384$
$d^{2}$
$(0.05)^{2}$

Where: $n=$ Sample size

$\mathrm{p}=$ Prevalence of herbal medicine use among pregnant women

$\mathrm{d}=$ Margin of sampling error tolerated

$Z=$ the standard normal value at confidence interval of $95 \%$

Since the total pregnant women attending ANC at WURH was less than 10,000 , which was 553 pregnant women, reduction formula was applied as follow;

$\mathrm{Nf}=$ 384
$(1+(n / N))$
$(1+(384 / 553))$

Where: $\quad \mathrm{Nf}=$ Final sample size

$\mathrm{n}=$ Calculated sample size

$\mathrm{N}=$ Source of population (553 pregnant women)

\section{Therefore, the final sample size was 226 pregnant women and adding $5 \%$ for non-response rate it becomes 238 . Then 238 pregnant women were recruited consequently and interviewed.}

\section{Data Collection Instrument and Procedure}

Data about socio-demographic, medical history and herbal medicine use of pregnant women was collected by semi-structured interview questionnaire items. The questionnaire was prepared in English language after reviewing varies related literatures. Then questionnaire was translated to Afan Oromo 
language and back translated to English to ensure consistency of meaning. Before beginning of the actual data collection, the questionnaire was pretested on $5 \%$ pregnant women attending Nekemte referral hospital antenatal care clinic and a slight modification was made on questionnaire.

\section{Data Processing and Analysis}

Data was coded, entered, and analyzed by using Statistical Program for the Social Sciences (SPSS) version 21 software. Univariate and Multivariate logistic regression analysis was used to determine factors associated with herbal medicine use. Association was significant when p-value is less than 0.05 .

\section{Ethical Consideration}

A formal letter was written from Wollega University, Institute of Health Sciences Ethical Review Committee. Permission was obtained from WURH in order to conduct the study. Written informed consent was obtained from study participants prior to data collection.

\section{Results}

\section{Socio-Demographic Characteristics of Study Participants}

In this study, 238 pregnant mothers were participated in the study. The respondents mean age was $31.3 \pm$ 5.64 years that range from 17 to 46 years. Protestant religious followers account 103 (43.28\%) of respondents and urban residents were 177 (74.37\%) of participants. Oromo ethnic group accounts 214 (89.92\%) of respondents. Majority of the respondents were married which account $233(97.90 \%)$ of participants. Sixty one (25.63\%) of participants were government employee while $146(61.34 \%)$ of the respondents income range from 1001 to 2000 Ethiopian Birr (ETB) per month. Also, about 83 (34.87\%) of participants had completed diploma/degree. A 147 (61.76\%) of participants live at distance less than five kilometer from the hospital.

Table 1: Socio-demographic characteristics of Pregnant Women Attending Antenatal Care at Wollega University Referral Hospital, 2018 


\begin{tabular}{|c|c|c|}
\hline Variables & Number (n) & Percent (\%) \\
\hline \multicolumn{3}{|l|}{ Age } \\
\hline $21-30$ years & 111 & 46.64 \\
\hline $31-40$ years & 93 & 39.07 \\
\hline$\geq 41$ years & 34 & 14.29 \\
\hline \multicolumn{3}{|l|}{ Ethnicity } \\
\hline Oromo & 214 & 89.92 \\
\hline Amhara & 18 & 7.56 \\
\hline Tigre & 3 & 1.26 \\
\hline Gurage & 3 & 1.26 \\
\hline \multicolumn{3}{|l|}{ Marital status } \\
\hline Single & 3 & 1.26 \\
\hline Married & 233 & 97.90 \\
\hline Widowed & 2 & 0.84 \\
\hline \multicolumn{3}{|l|}{ Religion } \\
\hline Protestant & 103 & 43.28 \\
\hline Orthodox & 65 & 27.31 \\
\hline Muslim & 48 & 20.17 \\
\hline Catholic & 15 & 6.30 \\
\hline Wakefata & 7 & 2.94 \\
\hline \multicolumn{3}{|l|}{ Educational status } \\
\hline Illiterates & 38 & 15.97 \\
\hline Primary & 31 & 13.02 \\
\hline Secondary & 35 & 14.71 \\
\hline College/university & 51 & 21.43 \\
\hline Diploma/degree & 83 & 34.87 \\
\hline \multicolumn{3}{|l|}{ Occupational status } \\
\hline Government employee & 61 & 25.63 \\
\hline Self-employee & 77 & 32.35 \\
\hline
\end{tabular}




\begin{tabular}{lll} 
Farmer & 27 & 11.34 \\
\hline House wife & 38 & 15.97 \\
\hline Student & 35 & 14.70 \\
\hline Monthly income, Ethiopian Birr (ETB) & & \\
\hline $1001-2000$ & 146 & 61.34 \\
\hline $2001-3000$ & 65 & 27.32 \\
\hline$>3000$ & 27 & 11.34 \\
\hline
\end{tabular}

\section{Place of residence}

$\begin{array}{lll}\text { Urban } & 177 & 74.37 \\ \text { Rural } & 61 & 25.63\end{array}$

\section{Distance from health facility}

\begin{tabular}{lcc}
\hline$<5 \mathrm{~km}$ & 147 & 61.76 \\
\hline $5-10 \mathrm{~km}$ & 44 & 18.49 \\
\hline$>10 \mathrm{~km}$ & 47 & 19.75 \\
\hline
\end{tabular}

\section{Number of children previously born}

\begin{tabular}{lll}
\hline No & 51 & 21.43 \\
\hline One & 50 & 21.01 \\
\hline Two & 63 & 26.47 \\
\hline Three & 48 & 20.12 \\
\hline >Four & 26 & 10.92
\end{tabular}

\section{Pregnancy related information and Herbal Medicine use practice}

One hundred twenty one (50.84\%) of respondents were at their second trimester of pregnancy stage. Most respondents, 220 (92.44\%) of them were first visited ANC at their first trimester of pregnancy. One hundred thirty (54.62\%) of respondents visited ANC less than four times. Two hundred seventeen $(91.18 \%)$ of participants has planned pregnancy whereas $204(85.71 \%)$ did not have history of adverse pregnancy outcome. One hundred fifty eight $(66.40 \%)$ of pregnant women used herbal medicine during current pregnancy. herbal medicine commonly used were garlic $58(24.37 \%)$ and ginger $43(18.07 \%)$ among participants. The most common indication to use herbal medicines was nausea/vomiting 65 (27.31\%) and headache $53(22.27 \%)$. About 49 (20.59\%) of pregnant mothers get herbal medicine from traditional healers/herbalist. Herbal medicine was used in among $81(34.03 \%)$ of respondents because 
"herbal medicine has fewer side effect". In 46 (19.33\%) of participants traditional healers serve as source of information about herbal medicine (table 2).

Table 2: Characteristics of herbal medicine use among Pregnant Women Attending Antenatal Care at Wollega University Referral Hospital, Nekemte, Ethiopia, 2018 
Herbal medication use

\begin{tabular}{|c|c|c|}
\hline Yes & 158 & 66.40 \\
\hline No & 80 & 33.6 \\
\hline \multicolumn{3}{|l|}{ Pregnancy stage } \\
\hline First Trimester & 65 & 27.31 \\
\hline Second Trimester & 121 & 50.84 \\
\hline Third Trimester & 52 & 21.85 \\
\hline \multicolumn{3}{|l|}{ Timing of first ANC } \\
\hline First Trimester & 220 & 92.44 \\
\hline Second Trimester & 18 & 7.56 \\
\hline \multicolumn{3}{|l|}{ Number of ANC visits } \\
\hline Less than four & 130 & 54.62 \\
\hline Four or more & 108 & 45.38 \\
\hline \multicolumn{3}{|l|}{ Current pregnancy } \\
\hline Planned & 217 & 91.18 \\
\hline Unplanned & 21 & 8.82 \\
\hline \multicolumn{3}{|l|}{ History of adverse pregnancy outcome } \\
\hline Yes & 34 & 14.29 \\
\hline No & 204 & 85.71 \\
\hline \multicolumn{3}{|l|}{ Reason for herbal medicine use } \\
\hline Herbal medicines are effective than convectional meds & 51 & 21.43 \\
\hline Herbal medicine have fewer side effects & 81 & 34.03 \\
\hline Herbal medicine are accessible without prescription & 26 & 10.92 \\
\hline \multicolumn{3}{|l|}{ For what purpose and ailments you used herbal medicine } \\
\hline Headache & 53 & 22.27 \\
\hline Nausea/vomiting & 65 & 27.31 \\
\hline Typhoid & 6 & 2.52 \\
\hline
\end{tabular}




\begin{tabular}{lll}
\hline Urinary tract infection & 5 & 2.10 \\
\hline Common cold & 22 & 9.24 \\
\hline Diarrhoea & 7 & 2.94 \\
\hline What types of herbs you have used & & \\
\hline Ginger & 43 & 18.07 \\
\hline Garlic & 58 & 24.37 \\
\hline Tenadam & 21 & 8.82 \\
\hline Tosign & 20 & 8.40 \\
\hline Demakese & 16 & 6.72 \\
\hline From where do you get these herbs & & \\
\hline Self-preparation & 49 & 20.59 \\
\hline Traditional healers/herbalist & 49 & 20.59 \\
\hline Traditional birth Attendant & 13 & 5.46 \\
\hline Neighbors & 18 & 7.56 \\
\hline Market place & 29 & 12.18 \\
\hline Source of information about herbal medicine & & \\
\hline Traditional healers & & 19.39 \\
\hline Health profession & & \\
\hline & & \\
\hline Religious leaders & & \\
\hline & & \\
\hline
\end{tabular}


Univariate logistic regression shows variables like monthly income, residence have significant association with herbal medicine use. However there was no significant association between herbal medication use and age group, education status, marital status, occupation status, number of children previously born, in both the univariate and multivariate logistic regression analysis.

In multivariate logistic regression, rural residence and low monthly income have a significant association to herbal medicine use. Planned pregnancy was inversely associated to herbal medicine use in multivariate logistic regression analysis. The herbal medicine use during pregnancy was 3.885 times more likely among rural residents ( $\mathrm{AOR}=3.885,95 \% \mathrm{Cl} 1.121-13.47$ ) as compared to urban residents. Similarly, Respondents who has monthly income between 1001 to 2000 ETB ( $A O R=2.585,95 \% \mathrm{Cl} 1.125-$ 5.940) were 2.585 times more likely to use Herbal medicine as compared to those who earn $>3000$ income. However, mothers of planned pregnancy ( $A O R=0.335,95 \% \mathrm{Cl} 0.114-0.986)$ were less likely to use Herbal medicine as compared to those who had unplanned pregnancy (table 3 ).

Table 3: Logistic regression analysis of factors associated with herbal medicine use among Pregnant Women Attending Antenatal Care at Wollega University Referral Hospital, Ethiopia, 2018. 
Variables
Herbal

medicine

use

Yes
Univariate analysis

P-Value
Multivariable analysis

AOR $(95 \% \mathrm{Cl})$

P.

value

\section{Education}

Illiterate

$27 \quad 11$

$0.683(0.298-$

0.369

$1.001(0.386-2.591)$

0.999

Primary school $\quad 22 \quad 9$

Secondary school

$22 \quad 13$

1.568)

0.409

$0.579(0.200-1.672)$

0.312

$0.686(0.281-$

1.139(0.428-3.037)

0.794

$35 \quad 16$

$52 \quad 31$

Diploma/degree

$1.0 \quad 1.0$

0.938

$0.959(0.334-2.753)$
1.0
0.983

$$
\text { 1.678) }
$$

0.991(0.438- 0.482

2.244)

0.767 (0.366-

1.607)

\section{Occupation}

Government

employee

Self-employee

Farmer

House wife

Student
$39 \quad 22$

$55 \quad 22$

$15 \quad 12$

$25 \quad 13$

$24 \quad 11$

1.0

0.251

0.985

0.759

$1.011(0.321-3.186)$

0.249

2.216(0.573-8.572)

0.752

$1.230(0.341-4.4280$

1.0

1.0

1.0

1.135(0.426-

3.020)

1.0

\section{Monthly income}

\begin{tabular}{|c|c|c|c|c|c|c|}
\hline $1001-2000$ & 53 & 93 & \multirow{3}{*}{$\begin{array}{l}3.159(1.465- \\
6.813) \\
1.467(0.917- \\
2.345) 1.0\end{array}$} & 0.0030 .110 & \multirow{3}{*}{$\begin{array}{l}2.585(1.125-5.940) \\
1.320(0.791-2.204) \\
1.0\end{array}$} & 0.025 \\
\hline $2001-3000$ & 25 & 40 & & \multirow[t]{2}{*}{1.0} & & 0.288 \\
\hline$\geq 3000$ & 15 & 12 & & & & 1.0 \\
\hline \multicolumn{7}{|l|}{ Residence } \\
\hline Urban & 110 & 67 & 1.0 & 1.0 & 1.0 & 1.0 \\
\hline Rural & 48 & 13 & $\begin{array}{l}2.249(1.135- \\
4.457)\end{array}$ & 0.02 & 3.885 (1.121-13.47) & 0.032 \\
\hline
\end{tabular}

\section{Distance from}

health facility

$<5 \mathrm{~km}$

$\begin{array}{ll}96 \quad 51 \quad & 1.739(0.817- \\ & 3.702\end{array}$

0.151

$0.669(0.166-2.689)$

0.571 


\begin{tabular}{lcccccc}
\hline $5-10 \mathrm{~km}$ & 26 & 18 & $\begin{array}{l}2.266(0.918- \\
5.595)\end{array}$ & 0.076 & $1.110(0.284-4.342)$ & 0.881 \\
\hline$>10 \mathrm{~km}$ & 36 & 11 & 1.0 & 1.0 & 1.0 & 1.0
\end{tabular}

\section{Pregnancy stage}

\begin{tabular}{|c|c|c|c|c|c|c|}
\hline First trimester & 47 & 18 & $\begin{array}{l}0.862(0.387- \\
1.920)\end{array}$ & 0.716 & $1.396(0.476-4.089)$ & 0.543 \\
\hline Second trimester & 75 & 46 & $\begin{array}{l}1.380(0.689- \\
2.762)\end{array}$ & 0.362 & $1.946(0.842-4.597)$ & 0.129 \\
\hline Third trimester & 36 & 16 & 1.0 & 1.0 & 1.0 & 1.0 \\
\hline \multicolumn{7}{|l|}{$\begin{array}{l}\text { Current pregnancy } \\
\text { status }\end{array}$} \\
\hline Planned & 147 & 70 & $\begin{array}{l}0.524(0.212- \\
1.291)\end{array}$ & 0.160 & $0.335(0.114-0.986)$ & 0.047 \\
\hline Unplanned & 11 & 10 & 1.0 & 1.0 & 1.0 & 1.0 \\
\hline \multicolumn{7}{|l|}{$\begin{array}{l}\text { Number of ANC } \\
\text { visit }\end{array}$} \\
\hline Less than four & 93 & 36 & $\begin{array}{l}0.585(0.339- \\
1.009)\end{array}$ & 0.054 & $0.502(0.244-1.035)$ & 0.62 \\
\hline$\geq$ four & 65 & 44 & 1.0 & 1.0 & 1.0 & 1.0 \\
\hline
\end{tabular}

\section{Time of $1^{\text {st }}$ ANC}

\begin{tabular}{|c|c|c|c|c|c|c|}
\hline First trimester & 148 & 72 & $\begin{array}{l}0.608(0.230- \\
1.606)\end{array}$ & 0.316 & $0.369(0.110-1.242)$ & 0.107 \\
\hline
\end{tabular}

Second trimester
History of Adverse
Drug reaction

$\begin{array}{lcccccc}\text { Yes } & 25 & 9 & 0.674(0.299- & 0.343 & 0.590(0.240-1.455) & 0.252 \\ & & & 1.523 & & & \\ \text { No } & 133 & 71 & 1.0 & 1.0 & 1.0 & 1.0\end{array}$

\section{Discussion}

The present study assessed herbal medicine use and its associated factors among pregnant women Attending Antenatal Care at Wollega University Referral Hospital, Nekemte, Ethiopia. The finding of this study showed $66.40 \%$ of respondents used herbal medicine during current pregnancy. This finding was in line with a studies conducted in public health facilities in Hossana Town, Southern Ethiopia (8). 
However, herbal medicine use among pregnant women is higher than a study conducted in Ghana were only $52.7 \%$, Nigeria $12 \%$, Uganda $20 \%$ and Iran $34.4 \%$ use herbal medicine during pregnancy $(9,10,13,15)$. This difference can be due to inter cultural and accessibility variance of herbal medicine across countries which influence their use. Also our current finding of herbal medicine use lower than a study conducted in Mali were 79.9.\% use herbal medicine (17). This variation might occurs due to disease prevalence between the study areas since malaria symptoms is common in Mali study which encourage use of medicinal plants to treat malaria.

This study showed that commonly used herbal medicines during current pregnancy was garlic 58 (24.37\%), ginger $43(18.07 \%)$, Tenadam $21(8.82 \%)$, tosign $20(8.40 \%)$ and Demakese $16(6.72 \%)$ which is in line with the findings of study conducted at Hossana town public health facilities, southern Ethiopia in which garlic (68.80\%), ginger (55.80\%), tenaadam (26.4\%), damakasse $(22.8 \%)$ and eucalyptus (11.6 $\%)$ were used among respondents (8). Herbal medicines were indicated for nausea/vomiting among 65 (27.31\%) and headache 53 (22.27\%) management among respondents which is similar with a study conducted in Iran and Kenya where women use herbal medicine for nausea/vomiting, back pain, toothache, indigestion and infectious diseases $(9,10)$.

Place of residence affected herbal medicine use of pregnant women. Pregnant women who live in rural area use herbal medicine more likely than urban area which is supported by a similar study conducted in Gondar, Ethiopia (4). The possible reasons for the high usage among rural residents might be due to availability of traditional medicine in rural area. Also studies supported mothers residing in the rural areas are generally more knowledgeable on Herbal Medicines when compared to mothers in the urban area (18). Also, Respondents who has monthly income between 1001 to 2000 ETB were 2.585 times more likely to use Herbal medicine as compared to those who earn $>3000$ ETB which is similar with the study done in Gondar Ethiopia (4). This can be due to cheap price of the herbal medicine relative to modern medicine services and low income participants favors use of traditional medicines $(19,20)$.

Mothers of planned pregnancy were less likely to use Herbal medicine as compared to those who had unplanned pregnancy. Unplanned pregnancy was associated with a significantly increased odds of Psychological Distress compared to planned pregnancy (21). Maternal emotions like embarrassment for getting pregnant and mother's own negative affection (22) can encourage the pregnant mothers to terminate pregnancy through illegal abortion by using herbal medicines.

\section{Limitation Of The Study}

Pregnant women might be confused or embarrassed to report the use of herbal medicines during data collection. Therefore, recall bias may influence the results.

\section{Conclusion}


The prevalence of herbal medicine use among pregnant women attending ANC at WURH was found to be $66.40 \%$. Commonly used herbs among pregnant women were ginger (Zingiber officinale) $43(18.07 \%)$ and Garlic 58 (24.37\%). The most common indication for use was nausea/vomiting 65 (27.31\%) and headache $53(22.27 \%)$. Rural residence and low income had significantly associated to herbal medicine use while planned pregnancy was negative associated to herbal medicine use.

\section{Declarations}

\section{Acknowledgements}

We would like to thank pregnant mothers attending antenatal care of Wollega University referral hospital. In addition, our thank goes to data collectors.

\section{Abbreviations and Acronyms}

AOR: adjusted odds ratio; ANC: Antenatal Care; COR: crude odds ratio; SPSS: Statistical Program for the Social Sciences; WURH: Wollega University Referral Hospital

\section{Authors' contributions}

BGL conceived and designed the study; extracted, analyzed and interpreted the data; and prepared manuscript. GFM, MGD, GBW, EAG and DDJ supervised the whole research, guided the conception and design of the study, and assisted with interpretation of data and manuscript preparation. All authors read and approved the final manuscript.

\section{Competing interests}

The authors declare that they have no competing interests.

\section{Availability of data and materials}

The datasets used and/or analyzed during the current study are available from the Principal investigator on reasonable request

\section{Consent for publication}

Not applicable.

\section{Ethical consideration}

Ethical clearance was obtained from Ethical Review Committee of Wollega University, institute of Health sciences. A formal letters were written to WURH in order to get permission to conduct the study. Participation in this study was on a voluntary basis and written consent was obtained from study subjects prior to data collection. 


\section{Funding}

There is no funding for this research

\section{References}

1. World Health Organization. Regional Office for the Western Pacific Manila, 1998. Guidelines for the appropriate use of herbal medicines. 1998.

2. Abdel-aziz SM, Aeron A, Kahil TA. Health Benefits and Possible Risks of Herbal Medicine. Microbes Food Heal. 2016;97-116.

3. John LJ, Shantakumari N. Herbal Medicines Use During Pregnancy: A Review from the Middle East. Oman Med J. 2015;30(4):229-36.

4. Mekuria AB, Erku DA, Gebresillassie BM, Birru EM, Tizazu B, Ahmedin A. Prevalence and associated factors of herbal medicine use among pregnant women on antenatal care follow-up at University of Gondar referral and teaching hospital , Ethiopia: a cross-sectional study. BMC Complement Altern Med. 2017;17(86):1-7.

5. Choi RJ, Wong AST, Jia W, Chang I-M, Wong RNS, Fan T-P, et al. The Art and Science of Traditional Medicine Part 3: The Global Impact of Traditional Medicine. 350 (6262). 2015. 54-56 p.

6. Oreagba IA, Oshikoya KA, Amachree M. Herbal medicine use among urban residents in Lagos, Nigeria. BMC Complement Altern Med. 2011;11(117):1-8.

7. Robinson MM, Zhang X. World Health organization. The World Medicines Situation 2011, Traditional medicines: Global situation, issues and challenges. In 2011. p. 1-12.

8. Laelago T, Yohannes T, Lemango F. Prevalence of herbal medicine use and associated factors among pregnant women attending antenatal care at public health facilities in Hossana Town , Southern Ethiopia: facility based cross sectional study. Arch Public Heal. 2016;74(7):1-8.

9. Tahery N, Mahmodi N, Shirzadegan R. The prevalence and causes of herbal drug use in pregnant women referring to Abadan health centers; a cross-sectional study in southwest Iran. J Prev Epidemiol. 2019;3(2):1-4.

10. Mothupi MC. Use of herbal medicine during pregnancy among women with access to public healthcare in Nairobi , Kenya: a cross-sectional survey. BMC Complement Altern Med. 2014;14(432):1-8.

11. Fatima N, Nayeem N. (October 26th 2016). Toxic Effects as a Result of Herbal Medicine Intake, Toxicology - New Aspects to This Scientific Conundrum, Sonia Soloneski and Marcelo L. Larramendy, IntechOpen.

12. Bepe N, Madanhi N, Mudzviti T, Gavi S, Maponga CC, Morse GD. The impact of herbal remedies on adverse effects and quality of life in HIV-infected individuals on antiretroviral therapy. $J$ Infect Dev Ctries. 2012;5(1):48-53. 
13. Ameade EPK, Zakaria AP, Abubakar L, Sandow R. Herbal medicine usage before and during pregnancy - a study in Northern Ghana. Int J Complement Altern Med. 2018;11(4):235-42.

14. Orief YI, Farghaly NF, Ibrahim MIA. Use of herbal medicines among pregnant women attending family health centers in Alexandria. Middle East Fertil Soc J. 2014;19:42-50.

15. Nyeko R, Tumwesigye NM, Halage AA. Prevalence and factors associated with use of herbal medicines during pregnancy among women attending postnatal clinics in Gulu district, Northern Uganda. BMC Pregnancy Childbirth. 2016;16(296):1-12.

16. Bayisa B, Tatiparthi R, Mulisa E. Use of Herbal Medicine Among Pregnant Women on Antenatal Care at Nekemte Hospital , Western Ethiopia. Jundishapur J Nat Pharm Prod. 2014;9(4):1-5.

17. Nergard CS, Ho TPT, Diallo D, Ballo N, Paulsen BS, Nordeng H. Attitudes and use of medicinal plants during pregnancy among women at health care centers in three regions of Mali, West-Africa. $J$ Ethnobiol Ethnomed. 2015;11(73):1-11.

18. Catublas HA. Knowledge, attitudes and practices in the use of herbal medicine: the case of urban and rural mothers in the philippines. Mahidol Univ J Pharm Sci. 2016;43(1):1-16.

19. Shewamene Z, Dune T, Smith CA. The use of traditional medicine in maternity care among African women in Africa and the diaspora: A systematic review. BMC Complement Altern Med. 2017;17(382):1-16.

20. Oyebode O, Kandala NB, Chilton PJ, Lilford RJ. Use of traditional medicine in middle-income countries: A WHO-SAGE study. Health Policy Plan. 2016;31(8):984-91.

21. Barton K, Redshaw M, Quigley MA, Carson C. Unplanned pregnancy and subsequent psychological distress in partnered women: A cross-sectional study of the role of relationship quality and wider social support. BMC Pregnancy Childbirth. 2017;17(44):1-9.

22. Akbarzadeh M, Yazdanpanahi Z, Zarshenas L, Sharif F. The Women'S Perceptions About Unwanted Pregnancy: A Qualitative Study in Iran. Glob J Health Sci. 2016;8(5):189-96. 